\title{
Polymorphism in the Assembly of Phase-Segregated Block Molecules: Pathway Control to 1D and 2D Nanostructures
}

\author{
Brigitte A. G. Lamers, ${ }^{\dagger}$ Robert Graf, ${ }^{\dagger}$ Bas F. M. de Waal, ${ }^{\dagger}$ Ghislaine Vantomme, ${ }^{\dagger}$ Anja R. A. Palmans, ${ }^{\dagger}$ \\ and E. W. Meijer*, ${ }^{\dagger}$ \\ ${ }^{\dagger}$ Institute for Complex Molecular Systems and Laboratory of Macromolecular and Organic Chemistry, Eindhoven University of \\ Technology, P.O. Box 513, 5600 MB Eindhoven, The Netherlands \\ ${ }^{\ddagger}$ Max Planck Institute for Polymer Research, Ackermannweg 10, 55128 Mainz, Germany \\ Supporting Information
}

\begin{abstract}
Nanomaterials with highly ordered, one- or twodimensional molecular morphologies have promising properties for adaptive materials. Here, we present the synthesis and structural characterization of dinitrohydrazone (hydz) functionalized oligodimethylsiloxanes ( $o$ DMSs) of discrete length, which form both 1- and 2D nanostructures by precisely controlling composition and temperature. The morphologies are highly ordered due to the discrete nature of the siloxane oligomers. Columnar, 1D structures are formed from the melt within a few seconds as a result of phase segregation in combination with $\pi-\pi$ stacking of the hydrazones. By tuning the length of the siloxane, the synergy between these

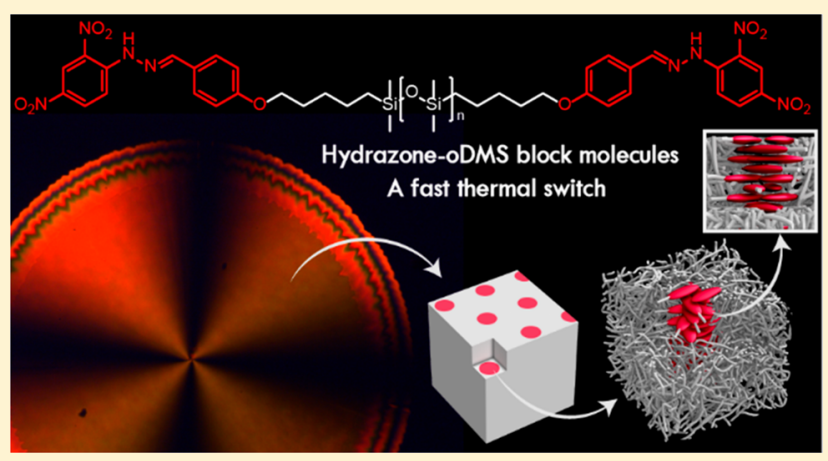
interactions is observed which results in a highly temperature sensitive material. Macroscopically, this gives a material that switches reversibly and fast between an ordered, solid and a disordered, liquid state at almost equal temperatures. Ordered, 2D lamellar structures are formed under thermodynamic control by cold crystallization of the hydrazones in the amorphous siloxane bulk via a slow process. We elucidate the 1- and 2D morphologies from the nanometer to molecular level by the combined use of solid state NMR and X-ray scattering. The exact packing of the hydrazone rods within the cylinders and lamellae surrounded the liquid-like siloxane matrix is clarified. These results demonstrate that controlling the assembly pathway in the bulk and with that, tuning the nanostructure dimensions and domain spacings, material properties are altered for applications in nanotechnology or thermoresponsive materials.
\end{abstract}

\section{INTRODUCTION}

Liquid crystals (LCs) and block copolymers (BCPs) form a variety of ordered structures that result in well-defined morphologies, which have been successfully applied in electronics as well as in optics or membranes. ${ }^{1-5}$ Insights into the relation between nanostructure morphology and macroscopic property is paramount to create functional materials for nanotechnology. Both LCs and BCPs can access the same type of morphologies such as lamellar, cylindrical, bicontinuous cubic, and spherical phases. ${ }^{6}$ However, the formation of ordered domains in $\mathrm{BCP}$ and $\mathrm{LC}$ materials proceeds via different mechanisms. For BCPs, phase segregation drives the morphology, which can be tuned by the BCP length $(N)$, composition, and Flory-Huggins interaction parameter $(\chi)^{7,8}$ In contrast, the nanostructure formation of LCs results from the directionality of the mesogens, i.e., rods or discs, in combination with the mobility of the side chains. ${ }^{9}$

A recent target in nanotechnology is to enhance long-range order and decrease domain spacings. This has resulted in fading boundaries between organic-LC and polymer-BCP chemistry by merging the directionality and crystallinity of
LCs with the phase segregation in BCPs. ${ }^{10}$ Early examples are phase-segregated LCs, while more recent examples are based on discrete length block co-oligomers. ${ }^{6,11-18}$ Herein, the absence of molar mass dispersity $(\nexists=1.00)$ results in phasesegregated structures even at very low degrees of polymerizations. ${ }^{17,19}$ Furthermore, the discrete length of the oligomers gives the ability to really design and understand structure formation into ordered morphologies. Inspired by telechelic molecules forming supramolecular polymers, ${ }^{20-25}$ we recently introduced oligodimethylsiloxane ( $o$ DMS) end-functionalized molecules. By balancing the volume fractions of the blocks and the directional group association, different molecular arrangements were formed based on supramolecular BCP phase segregation. ${ }^{26}$ The introduction of crystalline interactions next to phase segregation can further enhance the nanostructure organization. In $o$ DMS-based low MW amorphous-crystalline BCOs and block molecules with crystalline end-groups, the long-range order in the nanostructure morphology was improved indeed. ${ }^{26-31}$ However, due to the constraints

Received: August 13, 2019

Published: September 4, 2019 
imposed by the crystalline domains, the morphology is forced into a lamellar structure, irrespective of the volume fraction of the blocks. This crystallization driven self-assembly has been exploited for the formation of $2 \mathrm{D}$ nanomaterials. ${ }^{32,33}$

The directional interactions of discrete molecules are the driving force for self-assembly in the bulk and determine the final nanostructure morphology. An analogy can be made with the assembly of small molecules in solution since the directional interactions between molecules results in a variety of supramolecular morphologies, similar to phase-segregated structures in the bulk. Cylindrical, fibrous structures are formed through one-dimensional (1D) interactions, which can proceed via an isodesmic or cooperative/nucleation-elongation mechanism. ${ }^{34}$ Sheet-like architectures mostly assemble via crystallization of rigid molecules, hence formed via nucleation and growth. ${ }^{35-37}$ As a result of the mechanism by which they are formed and the pathway selection, the assembly of $1 \mathrm{D}$ or 2D nanostructures is highly dependent on environmental effects such as temperature and solvent. ${ }^{38,39}$ Structures formed under kinetic control can be different than those resulting from the thermodynamic equilibrium. ${ }^{40-44}$

Inspired by the pathway control in solution, we report here on the nanostructure formation of oDMS end-functionalized block molecules in the bulk into $1 \mathrm{D}$ and 2D morphologies. For this, we synthesized a novel family of homo- and heterotelechelic oDMS-based discrete oligomers end-capped with dinitrophenylhydrazones that can assemble either via crystallization or supramolecular interactions (Scheme 1). ${ }^{45,46}$

Scheme 1. Molecular Structures of hydz-Si ${ }_{15}$, hydz-Si $i_{n}-h y d z$, and hydz-Si $i_{\sim 22}$-hydz and Di- and Triblock Molecules ${ }^{a}$
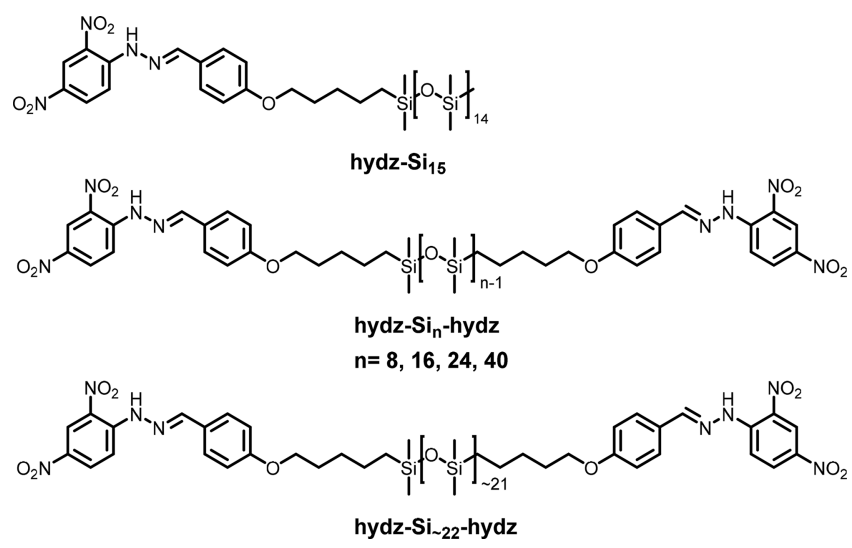

${ }^{a_{T}}$ The disperse block is indicated with a tilde $(\sim)$.

The liquid-like siloxane oligomer gives the molecules mobility and causes confinement of the hydrazones in an amorphous matrix. The compounds all show the presence of long-range, ordered domains. Solid state nuclear magnetic resonance spectroscopy in combination with $\mathrm{X}$-ray scattering gives detailed information on the polymorphism and driving forces of the hydrazone assembly in cylinders or lamellae. The morphology is controlled by thermodynamics and kinetics, and highly sensitive toward slight changes in temperature. This gives rise to fast thermal solid-liquid phase transitions in a very small temperature window, which can be utilized in thermoresponsive materials. Finally, we show the influence of dispersity by the incorporation of a disperse oDMS linker, which has not been reported for this type oDMS-based block molecules.

\section{RESULTS AND DISCUSSION}

Synthesis of Dinitrohydrazone-(oligo)dimethylsiloxane $\mathrm{Di}$ - and Triblock Molecules. We synthesized a series of $o$ DMS end-functionalized hydrazone triblock molecules and one diblock molecule (Scheme 1). Discrete $o$ DMS-dihydrides with chain lengths of exactly 8, 16, 24 , or 40 repeating units (denoted as $\mathrm{Si}_{8}, \mathrm{Si}_{16}, \mathrm{Si}_{24}$, or $\mathrm{Si}_{40}$ ) were synthesized via a stepwise procedure previously described by our group. ${ }^{26}$ Olefin terminated dinitrohydrazones (hydz) were attached to the $o$ DMS-dihydrides via a platinum catalyzed hydrosilylation reaction (Scheme S1). Similarly, a disperse oDMS triblock molecule $(\boxplus=1.2)$ was obtained from commercially available $o$ DMS-dihydride with an average of 22 siloxane repeating units (hydz-Si $\left.i_{\sim 22}-h y d z\right)$. Moreover, a diblock molecule (hydz-Si $\mathrm{i}_{15}$ ) was synthesized from discrete $o$ DMS-hydride containing only one reactive hydride group that was obtained via a literature procedure. ${ }^{16}$ All compounds were obtained in high yields (60-97\%) and molecular characterization by NMR, MS (Maldi-Tof), DSC, and GPC-SEC indicated the high purity of all of the materials (Figures S1S6).

Temperature Sensitivity of hydz-oDMS Di- and Triblock Molecules. Whereas all oDMS-dihydrides and oDMS-hydride are viscous fluids, after functionalization with the hydrazone group the block molecules appear as waxy solids. Under the polarized optical microscope (POM), the formation of birefringent structures was observed upon cooling from the melt (Figure 1). All discrete block molecules formed spherulites (Figure 1B-E), except the shortest analogue (hydz$\mathrm{Si}_{8}$-hydz), which formed a needle-like texture (Figure 1A). Interestingly, the hydz-Si ${ }_{16}$-hydz, hydz-Si ${ }_{24}$-hydz, and hydz-Si ${ }_{15}$ block molecules grow spherulites with sizes up to $5 \mathrm{~mm}$ from one nucleation point (Figure 1F). This has been reported
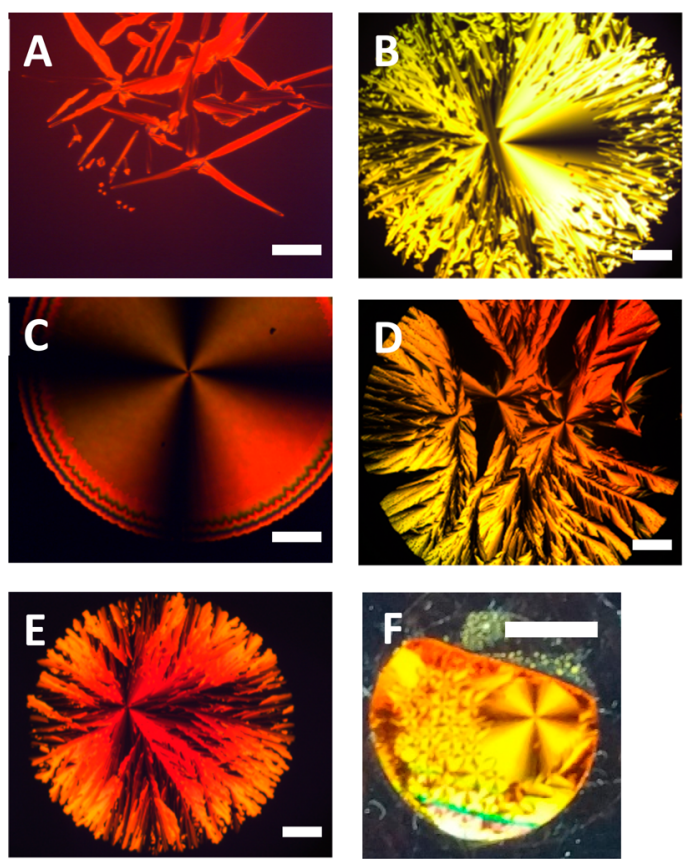

Figure 1. Polarized optical microscope images of hydz-Si $\mathrm{i}_{8}-\mathrm{hydz}(\mathrm{A})$, hydz-Si $i_{16}$-hydz (B), hydz-Si $i_{24}$-hydz (C), hydz-Si ${ }_{40}$-hydz (D), and hydz-Si ${ }_{15}$ (E). Scale bars represent $250 \mu \mathrm{m}$. Giant spherulite of hydz$\mathrm{Si}_{24}$-hydz under cross-polarizers (F), scale bar represents $5 \mathrm{~mm}$. Bulk material was melted between two glass plates and cooled at $2 \mathrm{~K} \mathrm{~min}^{-1}$. 
Table 1. Thermal Properties of hydz-oDMS Di- and Triblock Molecules

\begin{tabular}{|c|c|c|c|c|c|c|c|c|}
\hline entry & compound $^{a}$ & $M_{\mathrm{w}}^{b}\left[\mathrm{~g} \mathrm{~mol}^{-1}\right]$ & $T_{\mathrm{g}}^{c}\left[{ }^{\circ} \mathrm{C}\right]$ & $T_{1}^{d}\left[{ }^{\circ} \mathrm{C}\right]$ & $T_{2}{ }^{d}\left[{ }^{\circ} \mathrm{C}\right]$ & $\Delta H_{\mathrm{fus}}{ }^{d}\left[\mathrm{~kJ} \mathrm{~mol}^{-1}\right]$ & $T_{\mathrm{cc}}{ }^{d}\left[{ }^{\circ} \mathrm{C}\right]$ & $T_{\mathrm{m}}{ }^{d}\left[{ }^{\circ} \mathrm{C}\right]$ \\
\hline 1 & hydz-Si $i_{8}-h y d z$ & 1302.0 & 40.4 & 138.2 & 136.9 & 1.3 & 101.7 & 107.8 \\
\hline 2 & hydz-Si ${ }_{16}$-hydz & 1913.2 & 3.2 & 147.7 & 146.7 & 2.2 & 35.4 & 55.7 \\
\hline 3 & hydz-Si ${ }_{24}$-hydz & 2506.4 & -5.8 & 143.5 & 143.1 & 2.6 & n.o. & n.o. \\
\hline 4 & hydz-Si $i_{40}-$ hydz & 3692.9 & -12.6 & 117.3 & 116.0 & 3.9 & n.o. & n.o. \\
\hline 5 & hydz-Si $i_{15}$ & 1482.7 & -17.5 & 124.1 & 123.5 & 3.3 & n.o. & n.o. \\
\hline 6 & hydz-Si $i_{\sim 22}$-hydz & $\sim 2360$ & -8.5 & 115.4 & 112.8 & $\sim 1.1$ & 47.2 & 70.0 \\
\hline
\end{tabular}

${ }^{a}$ Block molecules hydz-Si $\mathrm{i}_{\mathrm{n}}$-hydz as depicted in Scheme 1. Disperse block is indicated with a tilde $(\sim) ;{ }^{b}$ Calculated molecular weight. ${ }^{c}$ Glass transition temperature $\left(T_{\mathrm{g}}\right)$ determined with DSC using a heating rate of $10 \mathrm{~K} \mathrm{~min}^{-1}$. ${ }^{d}$ Order-disorder temperature $\left(T_{1}\right)$ measured while heating, disorder-order temperature $\left(T_{2}\right)$ measured while cooling and enthalpy of fusion per mole block molecule measured while heating $\left(\Delta H_{\text {fus }}\right)$. Cold crystallization temperature $\left(T_{\mathrm{cc}}\right)$ and melting temperature $\left(T_{\mathrm{m}}\right)$ measured while heating. Determined with DSC using a heating and cooling rate of $2 \mathrm{~K} \mathrm{~min}^{-1}$. Enthalpic values are per mole of block molecule; n.o.: not observed.

occasionally for mixtures of polymers and small molecules, ${ }^{47,48}$ but for oligomers with covalently attached small molecule endgroups, such large textures have not been reported to date. The nucleation point for spherulite growth appeared on millisecond time scales, which gives a first hint that a very fast thermal phase transition occurs in these systems. The nucleation is always observed within the hole of the hot stage as this spot is slightly cooler than the surrounding material that is in direct contact with the hot stage. However, the location of the nucleation point within the hole varies upon repeatedly heating above the clearing temperature followed by cooling to the ordered structure. When the temperature was kept constant directly after nucleation, oscillations of the spherulite growth were observed (Video S1). These may result from the hot stage fluctuations causing small variations in temperature. Alternatively, the release of energy upon formation of the ordered structure may cause minor heating of the sample resulting in constantly clearing and growing of the structure. Hence, these block molecules are highly sensitive to minimal temperature changes around their transition temperatures.

We evaluated and quantified the thermal sensitivity around the transition temperatures by differential scanning calorimetry (DSC). Upon heating, all block molecules showed a glass transition $\left(T_{\mathrm{g}}\right)$ at temperatures below $40{ }^{\circ} \mathrm{C}$ (Table 1 , entry 1-5). For the discrete di- and triblock molecules, very sharp and narrow, endothermic peaks at temperatures between 117 and $147^{\circ} \mathrm{C}\left(T_{1}\right)$ were observed (Figure 2). The corresponding
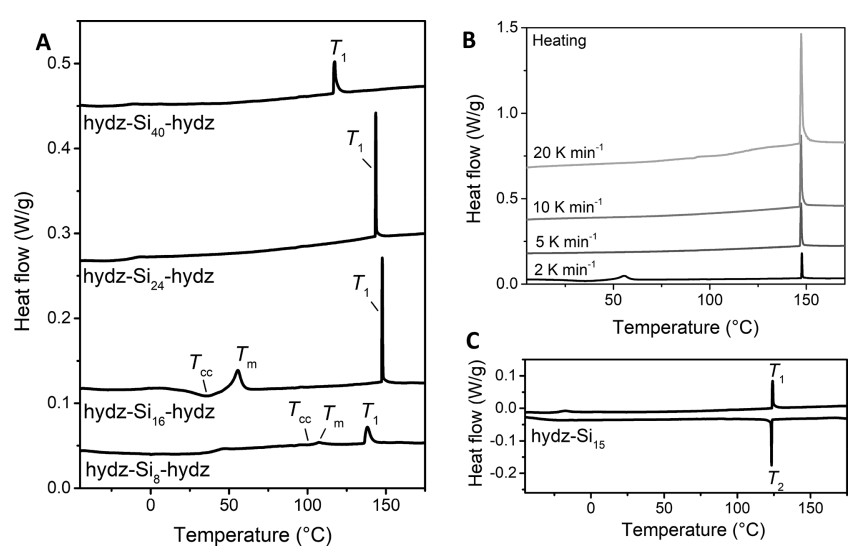

Figure 2. DSC traces (second heating run) of hydz-oDMS - hydz triblock molecules $(\mathrm{A})$ and hydz-Si $\mathrm{i}_{16}$-hydz with varying heating rates (B). DSC trace (second heating and cooling run) of hydz-Si ${ }_{15}$ diblock molecule (C). Endothermic heat flows have a positive value. A temperature ramp of $2 \mathrm{~K} \mathrm{~min}^{-1}$ was used if not otherwise specified. enthalpic energy $\left(\Delta H_{\mathrm{fus}}=1.3-3.9 \mathrm{~kJ} \mathrm{~mol}^{-1}\right)$ of this transition is relatively low compared to the crystalline hydrazone block $\left(\Delta H_{\text {fus }}=35.4 \mathrm{~kJ} \mathrm{~mol}^{-1}\right.$, Figure S7). Furthermore, the position of $T_{1}$ is independent of the heating rate (Figure $2 \mathrm{~B}$ ). Both results point to the presence of an order-disorder transition in the discrete systems. The related cooling traces of the materials revealed an equally sharp exothermic transition $T_{2}$ that is also independent of the cooling rate (Figure S8). Going through this transition, the material changed into a solid, ordered morphology. For all discrete block molecules, $T_{2}$ was nearly identical to the order-disorder transition $\left(T_{1}\right.$; Figures $2 \mathrm{C}$ and S9). In particular, these temperatures differ by only $0.4-1.3$ ${ }^{\circ} \mathrm{C}$, indicating that almost no supercooling is necessary to obtain the nanostructured material from the isotropic melt. Hence, the materials are extremely sensitive toward temperature fluctuations, as was observed at elevated temperatures under the POM.

Interestingly, for hydz-Si $\mathrm{i}_{8}$-hydz and hydz-Si $\mathrm{i}_{16}$-hydz two additional transitions were observed when a low heating rate was applied $\left(2 \mathrm{~K} \mathrm{~min}^{-1}\right.$, Figure $\left.2 \mathrm{~A}\right)$. With the help of variable temperature X-ray experiments (Figure S10), we could assign the lower temperature to a cold crystallization transition $\left(T_{\mathrm{cc}}\right)$ whereas the higher temperature is a melting transition $\left(T_{\mathrm{m}}\right)$. Both transitions are only observed when heating slowly $(2 \mathrm{~K}$ $\mathrm{min}^{-1}$ ) and disappear upon heating at $5 \mathrm{~K} \mathrm{~min}^{-1}$ or faster (Figure 2B). Hence, the formation of the crystalline phase for hydz-Si $i_{8}$-hydz and hydz-Si $i_{16}$-hydz is a slow process. The other structures with the longer siloxane-spacers did not show any crystallization.

Nanostructure Formation into 1D Assemblies. The nanoscale morphology of the hydrazone block molecules was examined using medium and wide-angle X-ray scattering experiments at room temperature. Well-defined and longrange ordered nanostructures were observed by the appearance of sharp reflection peaks (Figure 3A). All block molecules with $f_{\mathrm{Si}} \geq 0.72$ formed a hexagonally packed cylindrical phase (Table 2, entry 2-5), evidenced by regular scattering reflections at $q^{*}, \sqrt{ } 3 q^{*}, \sqrt{ } 4 q^{*}$, and $\sqrt{ } 7 q^{*}$ in the $1 \mathrm{D}$ transmission scattering profile (Figure $3 \mathrm{~A}$ ). In accordance to the microstructure observed by POM, the nanostructure of hydz-Si $i_{8}-h y d z$ was different from all the other discrete hydrazone block molecules. The scattering profile of the shortest analogue reveals a lamellar structure by the presence of integer multiples of the principle scattering peak ( $q^{*}$; Figure $3 \mathrm{~A})$. The domain spacings (d) range from 4.0 to $5.4 \mathrm{~nm}$ with increasing size of siloxane (Table 2 , entry $1-5$ ). The diblock molecule (hydz-Si $\mathrm{i}_{15}$ ) also forms hexagonally packed cylinders and its domain spacing matches perfectly the range of the 


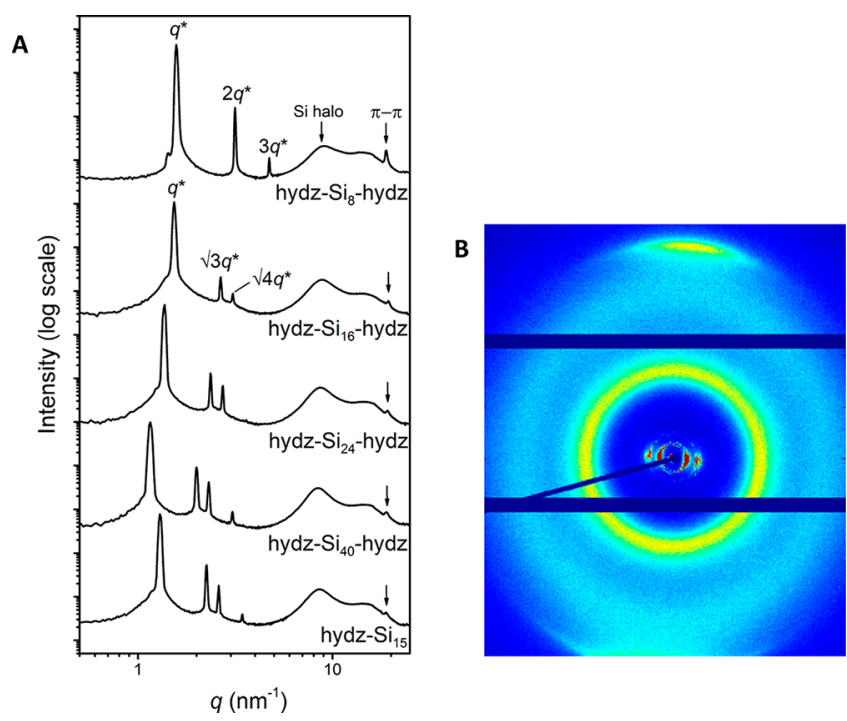

Figure 3. 1D transmission scattering profiles for hydz-oDMS tri- and diblock molecules at room temperature (A). 2D wide-angle $\mathrm{X}$-ray scattering profile for hydz-Si $\mathrm{i}_{16}$-hydz showing the alignment of the cylinders and directional $\pi-\pi$ stacking (B).

Table 2. Morphological Characterization of hydz-oDMS Diand Triblock Molecules

\begin{tabular}{|c|c|c|c|c|c|}
\hline entry & compound $^{a}$ & $f_{\mathrm{Si}}^{b}$ & phase $^{c}$ & $d^{c}[\mathrm{~nm}]$ & $d_{\text {cryst }}{ }^{d}[\mathrm{~nm}]$ \\
\hline 1 & hydz-Si $i_{8}-h y d z$ & 0.56 & LAM & 4.0 & 3.4 \\
\hline 2 & hydz-Si ${ }_{16}$-hydz & 0.72 & CYL & 4.2 & 4.9 \\
\hline 3 & hydz-Si ${ }_{24}-$ hydz & 0.80 & CYL & 4.6 & n.o. \\
\hline 4 & hydz-Si ${ }_{40}-$ hydz & 0.87 & CYL & 5.4 & n.o. \\
\hline 5 & hydz-Si $i_{15}$ & 0.83 & CYL & 4.9 & n.o. \\
\hline 6 & hydz-Si $i_{\sim 22}-$ hydz & $\sim 0.78$ & LAM & 5.6 & n.o. \\
\hline
\end{tabular}

${ }^{a}$ Block molecules hydz-Si ${ }_{\mathrm{n}}$-hydz as depicted in Scheme 1. Disperse block is indicated with a tide $(\sim) .{ }^{b}$ Volume fraction of the siloxane block, calculated using bulk densities for PDMS $\left(0.95 \mathrm{~g} \mathrm{~mL}^{-1}\right)^{16}$ and the crystal structure of 2,4-dinitrophenylhydrazone $\left(1.558 \mathrm{~g} \mathrm{~mL}^{-1}\right) .^{45}$ ${ }^{c}$ Morphology of nanostructure determined with SAXS at room temperature. $\mathrm{LAM}=$ lamellar phase. $\mathrm{CYL}=$ hexagonally packed cylinders. Domain spacing $(d)$ calculated using $d=2 \pi / q^{*}$. ${ }^{d}$ Domain spacing of crystalline, lamellar phase determined with SAXS at room temperature. Structure obtained upon annealing at 102 and $36{ }^{\circ} \mathrm{C}$ for hydz-Si $\mathrm{i}_{8}$-hydz and hydz-Si $\mathrm{i}_{16}$-hydz, respectively; n.o.: not observed.

triblock molecules considering the volume taken up by the siloxane fraction. Accordingly, we confirm similar packing models for the di- and triblock molecules in which the hydrazone block forms a cylinder with a diameter ranging from 2.4 to $2.7 \mathrm{~nm}$ (see SI for calculation).

A single reflection peak was observed in the wide angle region $\left(5<q<25 \mathrm{~nm}^{-1}\right)$ for all tri- and diblock molecules (Figure 3A). This represents a domain spacing of $0.3 \mathrm{~nm}$ which we assign to the interdisc distances, i.e., $\pi-\pi$ stacking of the aromatic rods. These interactions are highly directional when flow or shear alignment was applied to the sample, as shown in the $2 \mathrm{D} X$-ray scattering data for hydz-Si $i_{16}$-hydz (Figure 3B). Herein, the presence of highly aligned cylinders was observed and the direction of the interdisc interactions are found perpendicular to the cylinder orientation. The alignment of the hydrazones was further confirmed by measuring the absorbance of light linear and perpendicular to the column axis of shear aligned films. We found a dichroic ratio varying from 2.2 to 5.9 indicating that the absorption of light parallel to the columnar axis is lower than that perpendicular to the axis (Figure S11). Molecular orientation phenomena of the hydrazones in the cylindrical morphology were further studied by FT-IR. The presence of a $\nu(\mathrm{NH})$ peak at $3275 \mathrm{~nm}^{-1}$ indicates intramolecular hydrogen bonding as this peak did not disappear upon heating the sample to an isotropic state (Figure S12). A similar discotic hexagonal packing for semidiscoid dinitrophenylhydrazone molecules was reported by Paulus and co-workers. ${ }^{46}$ We share their conclusion and assign the formation of columnar structures to the antiparallel dimerization of the hydrazones occurring via dipole-dipole interactions.

Nanostructure Formation into 2D Assemblies under Thermodynamic Control. We measured variable temperature $\mathrm{X}$-ray scattering in order to elucidate the additional transitions $\left(T_{\mathrm{cc}}\right.$ and $\left.T_{\mathrm{m}}\right)$ for hydz-Si $\mathrm{i}_{8}-\mathrm{hydz}$ and hydz-Si $\mathrm{i}_{16}$-hydz observed by DSC upon heating at $2 \mathrm{~K} \mathrm{~min}^{-1}$. The morphology change of these block molecules was followed upon heating with the same rate. We observe a transition to a lamellar, crystalline structure for both block molecules when heated to $T_{\mathrm{cc}}$ (Figure S10). Heating above this temperature results in melting of the crystalline structure since melting is directly followed after crystallization, also observed by DSC (Figure $2 \mathrm{~A})$. However, we were able to trap the crystalline structure for hydz-Si $i_{8}$-hydz and hydz-Si $i_{16}$-hydz at room temperature by cooling directly after the $T_{\mathrm{cc}}$ was reached $\left(101.7\right.$ and $35.4{ }^{\circ} \mathrm{C}$, respectively). The resulting brittle solid consisted of a lamellar nanostructure for both block molecules, indicated by a principle scattering peak $\left(q^{*}\right)$ followed by its integer multiples in the $1 \mathrm{D}$ transmission scattering profile (Figure 4). Measuring

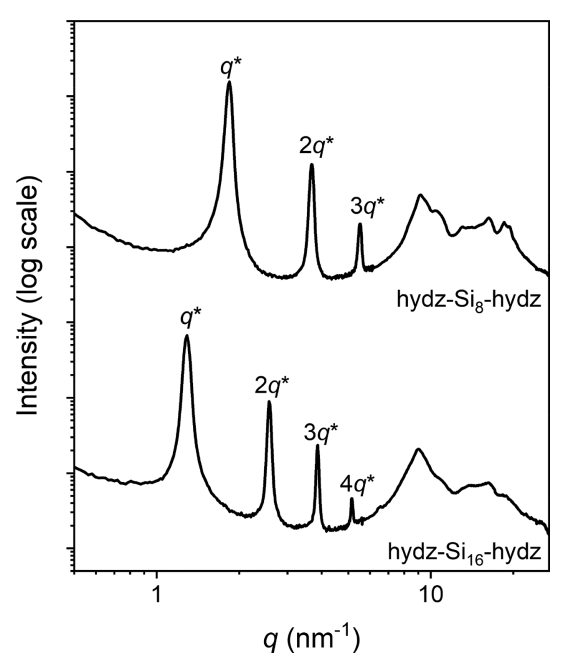

Figure 4. 1-D transmission scattering profiles of crystallized hydz-Si ${ }_{8}$ hydz and hydz-Si $i_{16}$-hydz, measured at room temperature.

in the wide-angle regime $\left(5<q<25 \mathrm{~nm}^{-1}\right)$ reveals crystallization of the hydrazone by the presence of multiple scattering peaks. The domain spacing of the lamellar, crystalline structure $\left(d_{\text {cryst }}\right)$ is 3.4 and $4.9 \mathrm{~nm}$ for hydz-Si $\mathrm{s}_{8}$ hydz and hydz-Si $i_{16}$-hydz, respectively (Table 2 , entry $1-2$ ). As a result of the crystallization, the lamellar domain spacing $\left(d_{\text {cryst }}\right)$ decreased by $0.6 \mathrm{~nm}$ compared to the amorphous lamellar domain spacing $(d=4.0 \mathrm{~nm})$ for hydz-Si $i_{8}$-hydz. Accordingly, the crystallized hydrazone rods are less shifted toward each other due to intermolecular hydrogen bonding (Figure S13). ${ }^{45}$ 
We have shown that the nanostructure of hydz-Si $i_{8}-h y d z$ and hydz-Si $i_{16}$-hydz can be tuned toward $1 \mathrm{D}$ or $2 \mathrm{D}$ assemblies by precisely controlling the temperature. The block molecules that contain a larger content of siloxane are stable 1D structures at room temperature up to the order-disorder temperature. Most likely, the siloxane content is too high to form crystalline structures and therefore the theoretical cold crystallization temperature would be far below room temperature. Hence, the long siloxanes give too much mobility to the molecular system in order to allow crystallization of the hydrazones, also explaining the extremely sharp disorderorder and order-disorder transitions.

Molecular Picture of the 1D and 2D Assemblies Revealed by Solid State NMR spectroscopy. Additional information on the molecular packing of the hydrazone rods was acquired using solid state NMR spectroscopy. The temperature-dependent ${ }^{1} \mathrm{H}$ and ${ }^{1} \mathrm{H}$ double-quantum (DQ)filtered MAS NMR spectra indicate that the siloxane is highly mobile at all temperatures (Figure S14 and S15). Thus, the amorphous siloxane can be seen as a bulk solvent for the hydrazone assembly, separating fibers of $\pi$-stacked hydrazone rods or layers of crystalline hydrazones. For the 1D assembly formed by hydz-Si $i_{24}$-hydz, the ${ }^{1} \mathrm{H}$ and ${ }^{1} \mathrm{H}$ DQ-filtered MAS NMR spectra also indicate that the $\alpha$-protons next to the ether experience the highest dynamic restrictions compared to the rest of the alkane spacer and siloxane oligomer (Figure S14). This means that this group finds itself at the interface of the hydrazone and siloxane phase. The packing of the $\pi$-stacked hydrazones in the cylinders was analyzed by the ${ }^{13} \mathrm{C}$ CP-MAS NMR spectra (Figure 5A). Herein, motionally broadened
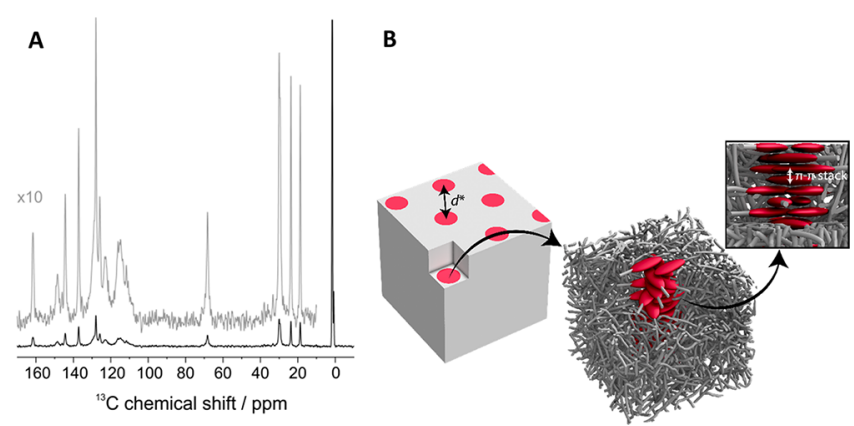

Figure 5. ${ }^{13} \mathrm{C}$ CP MAS NMR of cylinder forming hydz-Si $i_{24}$-hydz at 40 ${ }^{\circ} \mathrm{C}$ (A). Schematic representation of the proposed packing model of the cylinder-forming hydz-oDMS tri- and diblock molecules (B).

signals for the hydrazone units were observed, indicating substantial molecular fluctuations on the $10 \mathrm{kHz}$ to $\mathrm{MHz}$ time scale. The five sharp signals in the spectrum $(\delta>60 \mathrm{ppm})$ can be assigned to the quaternary aromatic sites in the hydrazone moiety. The chemical shifts of these signals are significantly less sensitive to local density fluctuations and molecular motions. Therefore, they show only a reduced motional broadening. Furthermore, the CP-MAS method, based on heteronuclear dipolar couplings, reveals that the hydrazone moieties do not move isotropically in the columnar phase. Combining these findings with the observed $\pi-\pi$ stacking reflection in the X-ray diffraction pattern leads to the structural model depicted in Figure 5B. Herein, the hydrazone units in the columns are stabilized by phase segregation and stack with a typical $\pi-\pi$ stacking distance of $0.3 \mathrm{~nm}$. The hydrazone pairs are randomly orientated, however, the central point of the column is the nitro-substituted phenyl ring that experiences the most restrictions in motion.

Moreover, the molecular packing of hydz-Si ${ }_{8}$-hydz and hydz$\mathrm{Si}_{16}$-hydz in the $2 \mathrm{D}$, crystalline morphology was obtained next to the packing into the $1 \mathrm{D}$ structure observed above $T_{\mathrm{m}}(60$ and $110{ }^{\circ} \mathrm{C}$, respectively). Insights into the crystalline packing of the hydrazones was obtained by the ${ }^{13} \mathrm{C}$ CP MAS NMR spectra. Herein, all peaks are sharp and the signal-to-noise ratio obtained under comparable experimental conditions as for the cylinder forming structures is significantly improved (Figure $6 \mathrm{~A})$. This indicates that in the $2 \mathrm{D}$ morphology, the hydrazone

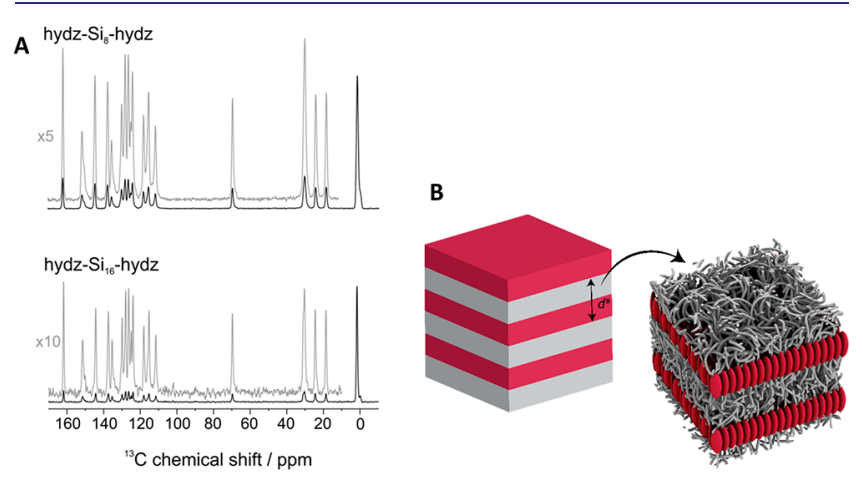

Figure $6 .{ }^{13} \mathrm{C}$ CP MAS NMR spectra of crystallized hydz-Si $i_{8}$-hydz and hydz-Si $i_{16}$-hydz, measured at $40{ }^{\circ} \mathrm{C}(\mathrm{A})$. Schematic representation of the proposed packing model of the crystalline lamellae-forming hydz$o$ DMS triblock molecules hydz-Si $i_{8}$-hydz and hydz-Si $i_{16}$-hydz (B).

units are fully immobilized in a crystalline environment within the amorphous, mobile siloxane matrix (Figure 6B). The sudden onset for line narrowing of the hydz-Si $i_{16}$-hydz and hydz-Si $i_{8}-$ hydz at the temperature just above $T_{\mathrm{m}}$ (60 and 110 ${ }^{\circ} \mathrm{C}$, respectively) in the ${ }^{1} \mathrm{H}$ MAS NMR confirms the crystalline state of the hydrazone units below these temperatures (Figure $\mathrm{S} 15$ ). Hence, the molecular mobility is increased rapidly above $T_{\mathrm{m}}$ while for the noncrystalline, $1 \mathrm{D}$ structure of hydz-Si $\mathrm{i}_{24}-\mathrm{hydz}$, the line narrowing was much more gradual in the same temperature range (Figure S14). The molecular packing of the hydz-Si $i_{8}$-hydz and hydz-Si $i_{16}$-hydz into the $1 \mathrm{D}$ structure observed above $T_{\mathrm{m}}$ is similar to the hydz- $\mathrm{Si}_{24}$-hydz described above.

Macroscopic Thermal Switching of hydz-oDMS Diand Triblock Molecules. The fast response toward temperature changes of the columnar structured materials shows that these materials have a great potential for a sensitive thermal switch. Thus, we evaluated the thermal switching between the liquid and the ordered, solid state macroscopically by rheology experiments over a temperature range of 100 to $160{ }^{\circ} \mathrm{C}$ (Figure 7). All discrete block molecules showed a sharp drop of the viscosity by almost 2 orders of magnitude within $8 \mathrm{~s}$ $\left(0.25{ }^{\circ} \mathrm{C}\right)$ upon heating the material (Figure S16). This drop appears at the order-disorder transition $\left(T_{1}\right)$ and corresponds to the solid/liquid transition. Upon cooling the sample, the material solidifies within the same time span and temperature. This result confirms and quantifies the narrow thermal transitions and the sensitivity toward thermal oscillations observed by DSC and POM, respectively. Such instantaneous drop in viscosity at the order-disorder transition is not reported to date for comparable low MW telechelic oligomers or previously reported block molecules. ${ }^{32}$

Effect of Dispersity on Nanostructure Morphology and Thermal Properties. In previous studies, we and others 


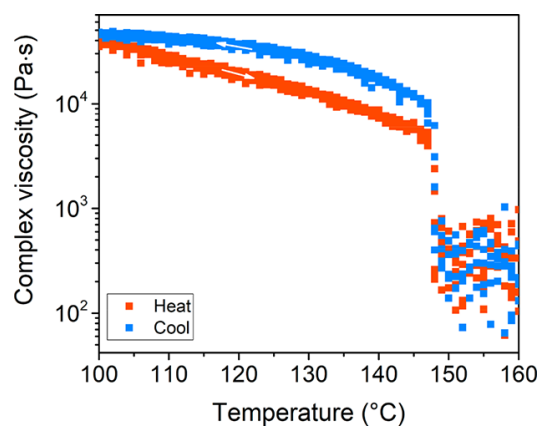

Figure 7. Complex viscosity change of hydz-Si $\mathrm{S}_{16}$-hydz measured upon heating (red) and cooling (blue) by rheology experiments. A constant strain of $0.01 \%$, angular frequency of $10 \mathrm{rad} \mathrm{s}^{-1}$ and a heating and cooling rate of $2 \mathrm{~K} \mathrm{~min}^{-1}$ were used. The viscosity values for the liquid region $\left(T>148{ }^{\circ} \mathrm{C}\right)$ have a high standard deviation as a result of melting of the block molecules.

have shown the effect of dispersity on morphologies formed by discrete length A-B BCOs. ${ }^{7,29,49,50}$ However, the consequences of dispersity in the $o$ DMS block in A-oDMS-A type triblock molecules has not been reported to date. The disperse hydz$\mathrm{Si}_{\sim 22}$-hydz block molecule $(\boxplus=1.2)$ with an average volume fraction siloxane of $\sim 0.78$ shows significant differences compared to its discrete counterpart hydz-Si $i_{24}$-hydz. First, the difference is visible under the POM by the appearance of an ill-defined structure (Figure 8A). X-ray scattering measure-
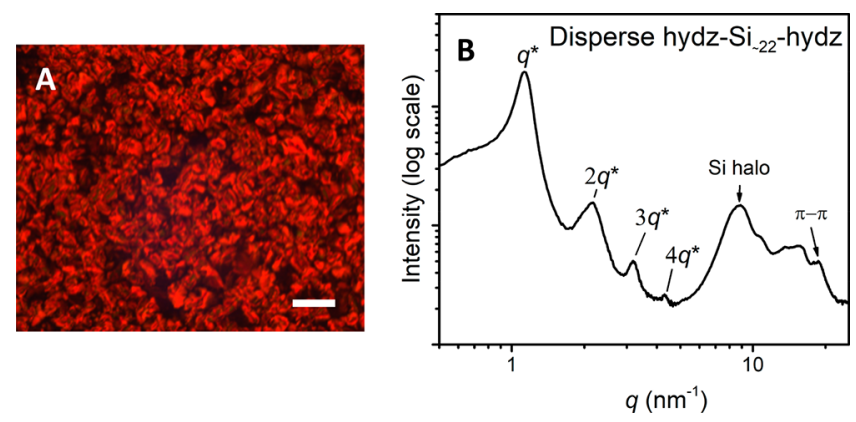

Figure 8. Polarized optical microscope image (A) and 1D transmission X-ray scattering profile for disperse hydz-Si $i_{\sim 22}$-hydz (B). The bulk material was melted between two glass plates and cooled down with $2 \mathrm{~K} \mathrm{~min}^{-1}$ for the microscope image. Scale bar represents $250 \mu \mathrm{m}$.

ments further highlight the disparity between the morphologies of discrete and the disperse compounds. For disperse hydz$\mathrm{Si}_{\sim 22}$-hydz, we can assign a lamellar phase by the presence of integer multiples of the principle scattering peak ( $q^{*}$; Figure $8 \mathrm{~B})$. The structure is somewhat distorted, as inferred by broadened reflection peaks and a shoulder on the low $q$-side of the principal scattering peak. Furthermore, some relatively weak scattering peaks are present in the higher $q$-region $(q>$ 5 ), indicating (partial) crystallization and the existence of $\pi-\pi$ stacking by the hydrazones in the lamellar matrix. As the discrete analogues form highly ordered cylinders (vide supra), the presence of dispersity results in significant changes in the morphology and dimensions of the nanostructures.

The introduction of dispersity in the hydrazone block molecule also results into the disappearance of the narrow transitions in DSC and with that, the fast thermal switching (Figure 9A). Upon heating, a cold crystallization transition temperature $\left(T_{\mathrm{cc}}\right)$ is observed followed by melting $\left(T_{\mathrm{m}}\right)$ similar
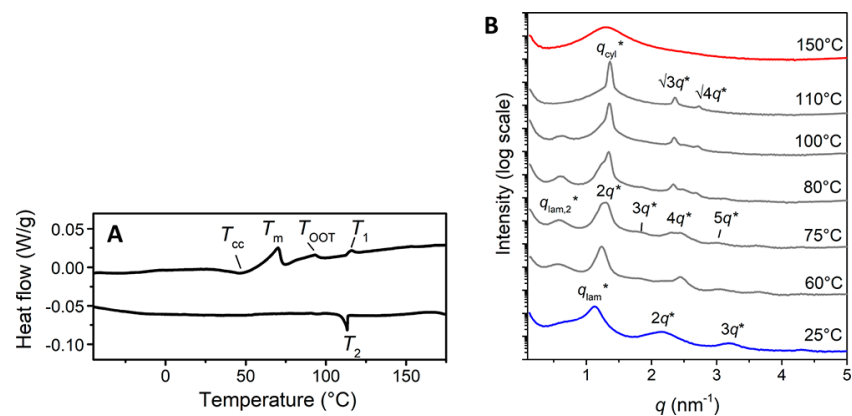

Figure 9. DSC trace (second heating and cooling run) (A) and variable temperature 1-D transmission X-ray scattering profiles (B) of the disperse hydz-Si $i_{\sim 22}$-hydz. Endothermic heat flows have a positive value. A temperature ramp of $2 \mathrm{~K} \mathrm{~min}^{-1}$ was used.

to the thermal transition in hydz-Si $i_{16}$-hydz. The melting is followed by two broad, endothermic transitions at 93.2 and $115.4{ }^{\circ} \mathrm{C}$. Variable temperature X-ray measurements show the nature of the transitions by changes in the morphology and domain spacing (Figure 9B). The first transition is a partial morphological change to another lamellar morphology with a larger domain spacing $\left(d_{\mathrm{lam}, 2}=10.5 \mathrm{~nm}\right)$, which is most likely due to the cold crystallization. Then, passing through the order-order temperature gives a morphology change from two lamellar phases to one hexagonally packed cylindrical phase with a domain spacing $\left(d_{\text {cyl }}\right)$ of $4.8 \mathrm{~nm}$. This is followed by the transition to a disordered liquid $\left(T_{1}\right)$. Most likely, the appearance of multiple morphological transitions is a result of various siloxane block lengths which becomes more important for low MW materials. Next to low MW BCOs, these results confirm the importance of a discrete design of block molecules to obtain the desired material properties and morphological precision.

\section{CONCLUSIONS}

We have presented the assembly of hydrazone-oDMS di- and triblock molecules with varying length of discrete siloxane oligomers. The hydz-oDMS-hydz triblock molecules organize in both a 1D and 2D nanostructure surrounded by the amorphous, liquid-like siloxane block, resembling solution selfassembly. The diblock molecule has similar thermal properties and nanostructure organization. The crystalline, 2D morphology is formed under thermodynamic control if the siloxane linker of the triblock molecules contains 8 or 16 repeating units. Immobilization of the hydrazone rod was observed, while the siloxane remained highly mobile. The 1D structure is formed rapidly over macroscopic length scales and irrespective of heating and cooling rates for all block molecules. The driving force for the $1 \mathrm{D}$ structure formation is phase segregation combined with $\pi-\pi$ stacking by dipole-dipole interactions. The structure is formed within a few seconds which we attribute to nucleation and elongation only occurring in one direction. The fast thermal response upon heating and cooling can be utilized in thermoresponsive materials. These materials can switch reversibly between the ordered, solid and amorphous, liquid state in a very efficient manner; almost no supercooling is necessary. The fastest thermal responses with the narrowest temperature range upon cooling and heating were found for the block molecules that have the complementary driving forces for 1D structure formation. Hence, the block molecules having volume fractions of siloxane matching a phase segregated hexagonally packed cylindrical 
phase combined with the hydrazone preferred assembly into $\pi$ stacked rods are the best thermal switchable material. Competing interactions which are observed for the longest and shortest triblock molecules, result in broader thermal transitions and decreased sensitivity toward temperature. Finally, we showed that there is a lack of morphologic control in the disperse triblock molecule in which a distorted, lamellar morphology was found at room temperature.

The presence of discrete siloxane linker between two assembling molecules is crucial to gain control over the nanostructure formation and obtain novel material properties. Tuning the exact length of the siloxane linker gives rise to various pathways of assembly favoring $1 \mathrm{D}$ or $2 \mathrm{D}$ nanostructures, which can be controlled by temperature. The sharp transition with a drop in viscosity opens new avenues to design materials with unparalleled ease of processing. Additionally, the discrete molecular design enabled us to find the synergy between two driving forces for structure formation and with that obtain the fastest and most sensitive thermoresponsive materials. Therefore, it is important to use discrete oligomers in the future to be able to design novel and highly responsive materials.

\section{ASSOCIATED CONTENT}

\section{S Supporting Information}

The Supporting Information is available free of charge on the ACS Publications website at DOI: 10.1021/jacs.9b08733.

Experimental procedures, characterization data, and Figures S1-S16 (PDF)

Video of the oscillations of the spherulite growth (MP4)

\section{AUTHOR INFORMATION}

\section{Corresponding Author}

*e.w.meijer@tue.nl

\section{ORCID $\odot$}

Ghislaine Vantomme: 0000-0003-2036-8892

Anja R. A. Palmans: 0000-0002-7201-1548

E. W. Meijer: 0000-0003-4126-7492

\section{Author Contributions}

The manuscript was written through contributions of all authors. All authors have given approval to the final version of the manuscript.

\section{Notes}

The authors declare no competing financial interest.

\section{ACKNOWLEDGMENTS}

The authors acknowledge Marcel van Genderen for the fruitful discussions. Ruth Cardinaels and Susana Petisco Ferrero are acknowledged for the help with the rheology measurements. We acknowledge funding from the Dutch Ministry of Education, Culture and Science (Gravity Program 024.001.035) and the Alexander von Humboldt Foundation.

\section{REFERENCES}

(1) Kato, T.; Uchida, J.; Ichikawa, T.; Sakamoto, T. Functional Liquid Crystals towards the Next Generation of Materials. Angew. Chem., Int. Ed. 2018, 57, 4355-4371.

(2) Cheng, J. Y.; Ross, C. A.; Smith, H. I.; Thomas, E. L. Templated Self-Assembly of Block Copolymers: Top-down Helps Bottom-Up. Adv. Mater. 2006, 18 (19), 2505-2521.

(3) Lugger, J.; Mulder, D.; Sijbesma, R.; Schenning, A. Nanoporous Polymers Based on Liquid Crystals. Materials 2018, 11, 104.
(4) Whitesides, G. M. Nanoscience, Nanotechnology, and Chemistry. Small 2005, 1 (2), 172-179.

(5) Schulze, B. M.; Shewmon, N. T.; Zhang, J.; Watkins, D. L.; Mudrick, J. P.; Cao, W.; Bou Zerdan, R.; Quartararo, A. J.; Ghiviriga, I.; Xue, J.; Castellano, R. K. Consequences of Hydrogen Bonding on Molecular Organization and Charge Transport in Molecular Organic Photovoltaic Materials. J. Mater. Chem. A 2014, 2 (5), 1541-1549.

(6) Tschierske, C. Non-Conventional Liquid Crystals-the Importance of Micro-Segregation for Self-Organisation. J. Mater. Chem. 1998, 8 (7), 1485-1508.

(7) Leibler, L. Theory of Microphase Separation in Block Copolymers. Macromolecules 1980, 13 (10), 1602-1617.

(8) Bates, C. M.; Bates, F. S. 50th Anniversary Perspective: Block Polymers-Pure Potential. Macromolecules 2017, 50 (1), 3-22.

(9) Demus, D.; Goodby, J.; Gray, G. W.; Spiess, H.-W.; Vill, V. Handbook of Liquid Crystals; Wiley-VCH: Weinheim, 1998.

(10) Hawker, C. J.; Wooley, K. L. The Convergence of Synthetic Organic and Polymer Chemistries. Science 2005, 309 (5738), 12001205.

(11) Newton, J.; Coles, H.; Hodge, P.; Hannington, J. Synthesis and Properties of Low-Molar-Mass Liquid-Crystalline Siloxane Derivatives. J. Mater. Chem. 1994, 4 (6), 869-874.

(12) Johansson, G.; Percec, V.; Ungar, G.; Smith, K. Fluorophobic Effect Generates a Systematic Approach to the Synthesis of the Simplest Class of Rodlike Liquid Crystals Containing a Single Benzene Unit. Chem. Mater. 1997, 9 (1), 164-175.

(13) Kato, T. Self-Assembly of Phase-Segregated Liquid Crystal Structures. Science 2002, 295 (5564), 2414-2418.

(14) Kato, T.; Mizoshita, N. Self-Assembly and Phase Segregation in Functional Liquid Crystals. Curr. Opin. Solid State Mater. Sci. 2002, 6 (6), 579-587.

(15) Nickmans, K.; Murphy, J. N.; Waal, B. De; Leclère, P.; Doise, J.; Gronheid, R.; Broer, D. J.; Schenning, A. P. H. J. Sub-5 Nm Patterning by Directed Self-Assembly of Oligo (Dimethylsiloxane) Liquid Crystal Thin Films. Adv. Mater. 2016, 28, 10068-10072.

(16) Van Genabeek, B.; de Waal, B. F. M.; Gosens, M. M. J.; Pitet, L. M.; Palmans, A. R. A.; Meijer, E. W. Synthesis and Self-Assembly of Discrete Dimethylsiloxane-Lactic Acid Diblock Co-Oligomers: The Dononacontamer and Its Shorter Homologues. J. Am. Chem. Soc. 2016, 138 (12), 4210-4218.

(17) Oschmann, B.; Lawrence, J.; Schulze, M. W.; Ren, J. M.; Anastasaki, A.; Luo, Y.; Nothling, M. D.; Pester, C. W.; Delaney, K. T.; Connal, L. A.; McGrath, A. J.; Clark, P. G.; Bates, C. M.; Hawker, C. J. Effects of Tailored Dispersity on the Self-Assembly of Dimethylsiloxane-Methyl Methacrylate Block Co-Oligomers. ACS Macro Lett. 2017, 6 (7), 668-673.

(18) Jiang, Y.; Golder, M. R.; Nguyen, H. V. T.; Wang, Y.; Zhong, M.; Barnes, J. C.; Ehrlich, D. J. C.; Johnson, J. A. Iterative Exponential Growth Synthesis and Assembly of Uniform Diblock Copolymers. J. Am. Chem. Soc. 2016, 138 (30), 9369-9372.

(19) van Genabeek, B.; de Waal, B. F. M.; Ligt, B.; Palmans, A. R. A.; Meijer, E. W. Dispersity under Scrutiny: Phase Behavior Differences between Disperse and Discrete Low Molecular Weight Block CoOligomers. ACS Macro Lett. 2017, 6 (7), 674-678.

(20) Fox, J. D.; Rowan, S. J. Supramolecular Polymerizations and Main-Chain Supramolecular Polymers. Macromolecules 2009, 42 (18), 6823-6835.

(21) Bouteiller, L. Hydrogen Bonded Polymers; Advances in Polymer Science, 2007; Vol. 207.

(22) Hirschberg, J. H. K. K.; Brunsveld, L.; Ramzi, A.; Vekemans, J. A. J. M.; Sijbesma, R. P.; Meijer, E. W. Helical Self-Assembled Polymers from Cooperative Stacking of Hydrogen-Bonded Pairs. Nature 2000, 407 (6801), 167-170.

(23) Van Beek, D. J. M.; Spiering, A. J. H.; Peters, G. W. M.; Te Nijenhuis, K.; Sijbesma, R. P. Unidirectional Dimerization and Stacking of Ureidopyrimidinone End Groups in Polycaprolactone Supramolecular Polymers. Macromolecules 2007, 40 (23), 8464-8475. 
(24) Müller, M.; Dardin, A.; Seidel, U.; Balsamo, V.; Iván, B.; Spiess, H. W.; Stadler, R. Junction Dynamics in Telechelic Hydrogen Bonded Polyisobutylene Networks. Macromolecules 1996, 29 (7), 2577-2583.

(25) Colombani, O.; Barioz, C.; Bouteiller, L.; Chanéac, C.; Fompérie, L.; Lortie, F.; Montés, H. Attempt toward 1D CrossLinked Thermoplastic Elastomers: Structure and Mechanical Properties of a New System. Macromolecules 2005, 38 (5), 1752-1759.

(26) Zha, R. H.; De Waal, B. F. M.; Lutz, M.; Teunissen, A. J. P.; Meijer, E. W. End Groups of Functionalized Siloxane Oligomers Direct Block-Copolymeric or Liquid-Crystalline Self-Assembly Behavior. J. Am. Chem. Soc. 2016, 138 (17), 5693-5698.

(27) Zha, R. H.; Vantomme, G.; Berrocal, J. A.; Gosens, R.; De Waal, B.; Meskers, S.; Meijer, E. W. Photoswitchable Nanomaterials Based on Hierarchically Organized Siloxane Oligomers. Adv. Funct. Mater. 2018, 28 (1), 1-8.

(28) Berrocal, J. A.; Zha, R. H.; de Waal, B. F. M.; Lugger, J. A. M.; Lutz, M.; Meijer, E. W. Unraveling the Driving Forces in the SelfAssembly of Monodisperse Naphthalenediimide-Oligodimethylsiloxane Block Molecules. ACS Nano 2017, 11 (4), 3733-3741.

(29) van Genabeek, B.; Lamers, B. A. G.; de Waal, B. F. M.; van Son, M. H. C.; Palmans, A. R. A.; Meijer, E. W. Amplifying (Im)perfection: The Impact of Crystallinity in Discrete and Disperse Block CoOligomers. J. Am. Chem. Soc. 2017, 139 (42), 14869-14872.

(30) Van Genabeek, B.; de Waal, B. F. M.; Palmans, A. R. A.; Meijer, E. W. Discrete Oligodimethylsiloxane-Oligomethylene $\mathrm{Di}-$ and Triblock Co-Oligomers: Synthesis and Self-Assembly and Molecular Organization. Polym. Chem. 2018, 9 (20), 2746-2758.

(31) Lamers, B. A. G.; van Genabeek, B.; Hennissen, J.; de Waal, B. F. M.; Palmans, A. R. A.; Meijer, E. W. Stereocomplexes of Discrete, Isotactic Lactic Acid Oligomers Conjugated with Oligodimethylsiloxanes. Macromolecules 2019, 52 (3), 1200-1209.

(32) Cortese, J.; Soulié-Ziakovic, C.; Cloitre, M.; Tencé-Girault, S.; Leibler, L. Order-Disorder Transition in Supramolecular Polymers. J. Am. Chem. Soc. 2011, 133 (49), 19672-19675.

(33) Ishiwari, F.; Okabe, G.; Ogiwara, H.; Kajitani, T.; Tokita, M.; Takata, M.; Fukushima, T. Terminal Functionalization with a Triptycene Motif That Dramatically Changes the Structural and Physical Properties of an Amorphous Polymer. J. Am. Chem. Soc. 2018, 140 (41), 13497-13502.

(34) De Greef, T. F. A.; Smulders, M. M. J.; Wolffs, M.; Schenning, A. P. H. J.; Sijbesma, R. P.; Meijer, E. W. Supramolecular Polymerization. Chem. Rev. 2009, 109 (11), 5687-5754.

(35) Vybornyi, M.; Rudnev, A.; Häner, R. Assembly of Extra-Large Nanosheets by Supramolecular Polymerization of Amphiphilic Pyrene Oligomers in Aqueous Solution. Chem. Mater. 2015, 27 (4), 14261431.

(36) Tidhar, Y.; Weissman, H.; Tworowski, D.; Rybtchinski, B. Mechanism of Crystalline Self-Assembly in Aqueous Medium: A Combined Cryo-TEM/kinetic Study. Chem. - Eur. J. 2014, 20 (33), 10332-10342.

(37) Avinash, M. B.; Govindaraju, T. Engineering Molecular Organization of Naphthalenediimides: Large Nanosheets with Metallic Conductivity and Attoliter Containers. Adv. Funct. Mater. 2011, 21 (20), 3875-3882.

(38) Ogi, S.; Stepanenko, V.; Sugiyasu, K.; Takeuchi, M.; Würthner, F. Mechanism of Self-Assembly Process and Seeded Supramolecular Polymerization of Perylene Bisimide Organogelator. J. Am. Chem. Soc. 2015, 137 (9), 3300-3307.

(39) Mabesoone, M. F. J.; Markvoort, A. J.; Banno, M.; Yamaguchi, T.; Helmich, F.; Naito, Y.; Yashima, E.; Palmans, A. R. A.; Meijer, E. W. Competing Interactions in Hierarchical Porphyrin Self-Assembly Introduce Robustness in Pathway Complexity. J. Am. Chem. Soc. 2018, 140 (25), 7810-7819.

(40) Fukui, T.; Kawai, S.; Fujinuma, S.; Matsushita, Y.; Yasuda, T.; Sakurai, T.; Seki, S.; Takeuchi, M.; Sugiyasu, K. Control over Differentiation of a Metastable Supramolecular Assembly in One and Two Dimensions. Nat. Chem. 2017, 9 (9), 493-499.
(41) Korevaar, P. A.; De Greef, T. F. A.; Meijer, E. W. Pathway Complexity in $\pi$-Conjugated Materials. Chem. Mater. 2014, 26 (1), 576-586.

(42) Rybtchinski, B. Adaptive Supramolecular Nanomaterials Based on Strong Noncovalent Interactions. ACS Nano 2011, 5 (9), 67916818.

(43) Tantakitti, F.; Boekhoven, J.; Wang, X.; Kazantsev, R. V.; Yu, T.; Li, J.; Zhuang, E.; Zandi, R.; Ortony, J. H.; Newcomb, C. J.; Palmer, L. C.; Shekhawat, G. S.; De La Cruz, M. O.; Schatz, G. C.; Stupp, S. I. Energy Landscapes and Functions of Supramolecular Systems. Nat. Mater. 2016, 15 (4), 469-476.

(44) Sorrenti, A.; Leira-Iglesias, J.; Markvoort, A. J.; De Greef, T. F. A.; Hermans, T. M. Non-Equilibrium Supramolecular Polymerization. Chem. Soc. Rev. 2017, 46 (18), 5476-5490.

(45) Szczęsna, B.; Urbańczyk-Lipkowska, Z. Cooperative Effect of Multiple Hydrogen Bonding Involving the Nitro Group: Solid State Dimeric Self-Assembly of O-, M- and P-Hydroxyphenyl-2,4Dinitrophenylhydrazones. New J. Chem. 2002, 26 (2), 243-249.

(46) Paulus, W.; Ringsdorf, H.; Diele, S.; Pelzl, G. Columnar Phases from Semi-Discoid Molecules: Phase Induction via Hydrogen Bonding and Charge Transfer Interactions. Liq. Cryst. 1991, 9 (6), $807-819$.

(47) Wu, Q.-Y.; Wan, L.-S.; Xu, Z.-K. Centimeter-Scale Giant Spherulites in Mixtures of Polar Polymers and Crystallizable Diluents: Morphology, Structure, Formation and Application. RSC Adv. 2013, 3 (38), 17105.

(48) Rahman, M. H.; Nandi, A. K. Supramolecular Organization of Poly(vinylidene Fluoride)-Camphor Sulfonic Acid Blends: Giant Spherulites. Langmuir 2003, 19 (17), 7056-7060.

(49) Van Genabeek, B.; De Waal, B. F. M.; Ligt, B.; Palmans, A. R. A.; Meijer, E. W. Dispersity under Scrutiny: Phase Behavior Differences between Disperse and Discrete Low Molecular Weight Block Co-Oligomers. ACS Macro Lett. 2017, 6 (7), 674-678.

(50) Gangloff, N.; Höferth, M.; Stepanenko, V.; Sochor, B.; Schummer, B.; Nickel, J.; Walles, H.; Hanke, R.; Würthner, F.; Zuckermann, R. N.; Luxenhofer, R. Linking Two Worlds in Polymer Chemistry: The Influence of Block Uniformity and Dispersity in Amphiphilic Block Copolypeptoids on Their Self-Assembly. Biopolymers 2019, 110 (4), e23259. 